\title{
Factores asociados a mortalidad en enfermedad SARS-CoV-2 grave de un hospital
} peruano

\section{Factors associated with mortality in severe SARS-CoV-2 infection in a Peruvian hospital}

https://doi.org/10.35434/rcmhnaaa.2021.143.1245

\section{RESUMEN}

Objetivo: Determinar factores asociados a mortalidad en pacientes con enfermedad grave por SARS-CoV-2. Material y métodos: Estudio retrospectivo en pacientes mayores de 18 años hospitalizados por enfermedad grave (saturación periférica de Oxígeno < $93 \%$ o compromiso radiológico pulmonar $>30 \%$ ) y prueba molecular positiva en hisopado nasofaríngeo; en el hospital Rebagliati entre marzo y mayo de 2020. Se revisó historia clínica electrónica, resultados de análisis (de laboratorio y radiológicos) al ingreso y tratamiento recibido. Se realizó estadística bivariada y multivariada utilizando regresión de cox. Resultados: Se incluyeron 337 pacientes, de los cuales 215 fallecieron (63.8\%). Fueron de sexo masculino $72.1 \%$, edad promedio 63.6 años (DS 15.3), tiempo de enfermedad 7.4 días, estancia hospitalaria 10.3 días y $48.8 \%$ ingresó a ventilación mecánica invasiva. El análisis multivariado encontró significancia en edad > 60 años (HR 1.76, IC95\% 1.262.45), antecedente de diabetes mellitus tipo 2 (HR 1.63, IC95\% 1.14-2.32), compromiso radiológico pulmonar > $75 \%$ (HR 1.87, IC95\% 1.37-2.56), leucocitosis > $14000 x$ mm3 (HR 1.59, IC95\% 1.15-2.20), albúmina sérica < 4.0 $\mathrm{g} / \mathrm{dL}$ (HR 2.49, IC95\% 1.30-4.75) y lactato > $1.5 \mathrm{mmol} / \mathrm{L}$ (HR: 1.766 IC95\% 1.25-2.49). Conclusiones: La edad mayor de 60 años, diabetes mellitus tipo 2, compromiso pulmonar severo, leucocitosis, albúmina baja y lactato elevado al ingreso hospitalario, son factores asociados a mortalidad en pacientes con enfermedad grave por SARS-CoV-2.

Palabras Clave: Factores de riesgo; mortalidad hospitalaria; SARS-CoV-2; COVID-19; infección por coronavirus (Fuente: DeCS-BIREME).

\section{ABSTRACT}

Objetive: To determine factors associated with mortality in patients with severe SARS-CoV-2 disease. Materials and methods: Retrospective study in
FILIACIÓN

1. Facultad de Medicina Universidad Nacional Mayor de San Marcos, Lima, Perú

2. Hospital Edgardo Rebagliati Martins, EsSalud, Lima, Perú.

3. Centro de Investigación de Epidemiología Clínica y Medicina Basada en Evidencia, Universidad San Martín de Porres, Lima, Perú.

4. Universidad San Martín de Porres, Lima, Perú.

5. Clínica Delgado, Lima, Perú.

6. Instituto de Evaluación de Tecnología Sanitaria e Investigación, IETSI, EsSalud Perú.

7. Unidad de revisiones sistemáticas y metaanálisis, guías de práctica clínica y evaluaciones tecnológicas sanitarias. Universidad San Ignacio de Loyola, Lima, Perú.

a. Doctor en medicina.

b. Médico internista.

c. Médico residente.

e. Médico especialista en epidemiología.

f. Médico especialista en Medicina de Emergencias y Desastres.

g. Médico cardiólogo.

ORCID

1. JoséAmado-Tineo / 0000-0002-3286-4650

2. Ricardo Ayala-García / 0000-0002-7913-8728

3. Moises Apolaya-Segura / 0000-0001-5650-9998

4. Roxana Mamani-Quiroz / $\frac{0000-0002-9725-5565}{0000-0002-4059-0637}$

5. Javier Matta-Pérez / $\frac{0000-0002-4059-0637}{6 .}$

6. Germán Valenzuela-Rodríguez / 0000-0002-8595-

7. Angel Ardiles-Melgarejo / $\frac{0000-0002-5727-7233}{8 .}$ Carlos Marcos-Hernández / 0000-0001-5825-9080

8. Carlos Marcos-Hernández / 0000-0001-5825-9080

9. Waldo Taype-Huamaní / 0000-0002-8559-2513

11. Sthephany Matos-Santiváñez / 0000-0002-7897-4409

12. Loyda Miranda-Chávez / 0000-0002-0912-3598

13. Teodoro Oscanoa-Espinoza / 0000-0001-9379-4767

CORRESPONDENCIA

JoséAmado-Tineo

EMAIL

jpamadot@gmail.com

CONFLICTOS DE INTERÉS

Los autores niegan conflictos de interés.

\section{FINANCIAMIENTO}

Autofinanciamiento.

\section{REVISIÓN DE PARES}

Recibido: $15 / 05 / 2021$

Aceptado: 21/08/2021

\section{COMO CITAR}

Amado-Tineo, J., Ayala-García, R., Apolaya-Segura, M., Mamani-Quiroz, R., Matta-Pérez, J. Valenzuela-Rodríguez, G., Ardiles-Melgarejo, A., Marcos-Hernández, C., Taype-Huamaní, W. Rojas-Guimaray, J., Matos-Santiváñez, S., Miranda-Chávez, L., \& Oscanoa-Espinoza, T. Factores asociados a mortalidad en enfermedad SARS-CoV-2 grave de un hospital peruano. Revista Del Cuerpo Médico Hospital Nacional Almanzor Aguinaga Asenjo, 2021, 14(3), 280 286. https://doi.org/10.35434/rcmhnaaa.2021.143.1245

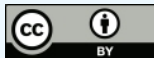

Esta obra está bajo una Licencia Creative Commons Atribución 4.0 Internacional. Versión Impresa: ISSN: 2225-5109

Versión Electrónica: ISSN: 2227-4731

Cross Ref. DOI: $10.35434 /$ rcmhnaaa

OJS: https://cmhnaaa.org.pe/ojs 
patients older than 18 years hospitalized with severe disease (peripheral oxygen saturation <93\% or radiological lung involvement $>30 \%$ ) and a positive RT-PCR nasopharyngeal swab; in the Rebagliati hospital between March and May 2020. We reviewed electronic medical record, results of admission studies (laboratory and radiological) and treatment received. Bivariate and multivariate analysis were performed using cox regression. Results: 337 patients were studied, 215 of them died (63.8\%). 72.1\% were male, mean age was 63.6 years (SD 15.3), time of disease evolution was 7.4 days, hospital stay 10.3 days, and $48.8 \%$ were on mechanical ventilation. Multivariate analysis found significance in age $>60$ years (HR 1.76 , IC95\% 1.26-2.45), history of type 2 Diabetes (HR 1.63, IC95\% 1.14-2.32), pulmonary involvement > 75\% (HR 1.87, IC95\% 1.372.56), leukocytes > $14000 \times \mathrm{mm} 3$ (HR 1.59, IC95\% 1.15-2.20), serum albumin < $4.0 \mathrm{~g} / \mathrm{dL}$ (HR 2.49, IC95\% 1.30-4.75) and lactate > $1.5 \mathrm{mmol} / \mathrm{L}$ (HR: 1.766 IC95\% 1.25-2.49). Conclusions: Age older than 60 years, type 2 diabetes mellitus, severe pulmonary involvement, leukocytosis, low albumin, and high lactate at admission are factors associated with mortality in patients with severe SARS-CoV-2 disease.

Keywords: Risk Factors; Hospital Mortality; SARS-CoV-2; COVID-19; Coronavirus Infections. (Source: DeCS-BIREME).

\section{INTRODUCCION}

En China, a fines del 2019, se reportaron casos graves de neumonía, identificando como agente causante un nuevo virus denominado Severe Acute Respiratory Syndrome CoronaVirus 2 (SARS-CoV-2). Esta enfermedad fue denominada COVID-19 (del inglés Coronavirus Disease 2019), de presunto origen zoonótico y con la capacidad de propagarse de persona a persona, hasta ser declarada pandemia por la Organización Mundial de la Salud el 11 de marzo de $2020^{(1-3)}$.

China (específicamente Wuham-Hubei) fue el primer foco de infección, notificándose más de 80 mil casos confirmados y una tasa de letalidad de 5,3\%. En marzo del mismo año se diseminó en Europa, siendo Italia y España los países más afectados, con 13,6 y $7,0 \%$ de casos fatales respectivamente (61,8 y 58,3 muertos por 100 mil habitantes). En el mes de Abril el foco de la pandemia se trasladó a América, siendo Estados Unidos de Norteamérica, Brasil, México y Perú los países más afectados. Para noviembre de 2020 en Argentina y Colombia se han incrementado significativamente los infectados y fallecidos por SARS-CoV-2 y en Europa se registra una segunda ola de contagios, mayor a la primera ${ }^{(3,4)}$.

En Perú se confirmó el primer caso el 6 de marzo de 2020 y los primeros fallecimientos el 19 del mismo mes, incrementándose rápidamente el número de casos, en los siguientes 4 meses se constituyó en uno de los países más afectados por la pandemia en el mundo. Hasta el 12 de Noviembre se han reportado 930 mil casos y 35067 fallecidos (tasa de letalidad de casos 3,77\% y 109.5 muertes por 100 mil habitantes) ${ }^{(4-6)}$.

La forma grave de COVID-19 se presenta en aproximadamente el $20 \%$ de los infectados y el $5 \%$ desarrolla síndrome de distrés respiratorio agudo y necesita cuidados intensivos y soporte ventilatorio avanzado ${ }^{(1,7-9)}$. Se han identificado factores de riesgo para desarrollo de la forma grave de COVID-19 y mayor riesgo de mortalidad, entre los que destacan el género masculino, edad avanzada, hipertensión arterial (HTA), obesidad, diabetes mellitus, enfermedades respiratorias y/o cardiovasculares crónicas. Reportándose también como factores asociados a severidad diversas manifestaciones clínicas, resultados de laboratorio y hallazgos radiológicos ${ }^{(10-13)}$.
El hospital nacional Edgardo Rebagliati Martins, perteneciente al Seguro Social de Salud (EsSalud), es considerado uno de los centros de referencia para COVID-19 en Perú y viene atendiendo un importante número de pacientes hospitalizados con alto porcentaje de fallecimientos y necesidad de soporte ventilatorio ${ }^{(14)}$. Por lo que el presente estudio busca determinar los factores (epidemiológicos, clínicos, de exámenes auxiliares y tratamiento) asociados a mortalidad en pacientes con enfermedad grave por SARS-Cov-2 en un hospital terciario de Lima.

\section{MATERIALES Y MÉTODOS}

La investigación fue desarrollada en el Hospital Nacional Edgardo Rebagliati Martins, ubicado en el distrito de Jesús María en Lima-Perú. Hospital de referencia de EsSalud, con 1600 camas hospitalarias que adecuó su oferta para recibir en sus dos terceras partes a pacientes con COVID-19.

Se realizó un estudio observacional retrospectivo, en pacientes hospitalizados entre marzo y mayo del 2020, con diagnóstico de COVID-19 clasificado como enfermedad grave. Todos los pacientes fueron confirmados por pruebas TR-PCR (reacción en cadena de polimerasa con transcriptasa reversa a tiempo real) para SARS-CoV-2.

Se calculó un tamaño de muestra de 149 participantes por cada grupo, considerando un reporte previo donde se reporta que de los pacientes graves (desarrollaron SDRA o fallecieron) el $27 \%$ tenían HTA, en comparación al $13 \%$ de los que no tuvieron esta condición ${ }^{(11)}$, proporción de perdidas $10 \%$, razón entre grupos $1: 1$, potencia $90 \%$ y con un nivel de confianza de $95 \%$.

Los criterios de inclusión fueron edad mayor de 18 años, TRPCR positiva y enfermedad grave por COVID-19 (Saturación periférica de Oxígeno previo al inicio de oxigenoterapia menor a $93 \%$ o compromiso pulmonar $>30 \%$ en la tomografía pulmonar). Se excluyeron a los pacientes en los cuales no se logró conocer su evento final (referidos o altas voluntarias), causa de fallecimiento no relacionada a COVID-19 y pacientes que tuvieron seguimiento incompleto.

Se revisaron los datos de la historia clínica electrónica de los pacientes, sin realizar ninguna modificación de los procesos de atención del hospital. Se consideraron factores 
epidemiológicos: edad, sexo, comorbilidades previas como HTA, diabetes, obesidad o enfermedad pulmonar crónica. Entre los factores clínicos se revisó tiempo de enfermedad, disnea, saturación al ingreso y qSOFA (del inglés quick Sequential Organ Failure Assessment). Entre los exámenes auxiliares al ingreso hospitalario se evaluaron recuento de leucocitos, monocitos, linfocitos, proteína $C$ reactiva (PCR), transaminasas, deshidrogenasa láctica $(\mathrm{DHL})$, dímero $\mathrm{D}$, fibrinógeno, análisis de gases arteriales, patrones radiológicos pulmonares y porcentaje de compromiso pulmonar según total severity score $(\mathrm{TSS})^{(13)}$ (en tomografía espiral multicorte sin contraste o radiografía simple de tórax). Entre los factores de tratamiento se evaluó medicación recibida por el paciente durante su hospitalización (de duración mayor a 48 horas), agrupándolos en: "fármacos contra COVID-19" recomendados en el país al momento de realizar el estudio (azitromicina, hidroxicloroquina e ivermectina), uso de corticoides, anticoagulación y ventilación mecánica (VM) invasiva. Se consideró como evento de interés al paciente fallecido, y como variable de tiempo a los días de estancia hospitalaria.

Los datos fueron codificados y procesados en SPSS versión 25.0 realizándose un análisis descriptivo de las variables estudiadas, utilizando prueba de Kolmogorov-Smirnov para evaluar la distribución normal. Además, se calcularon frecuencias, medidas de tendencia central y medidas de dispersión. En el análisis bivariado se determinaron los Hazard ratio (HR) de los factores asociados a mortalidad utilizando modelos de regresión de cox, y para el análisis multivariado se aplicó regresión de cox con método de selección hacia adelante condicional, ingresando al modelo estadístico aquellas variables cuyo valor $\mathrm{p}$ sea menor a 0,20 y/o tengan significancia clínica.

El proyecto fue autorizado por el Departamento de Emergencia del hospital y aprobado por comité de Ética en Investigación para COVID 19 de EsSalud (ÉTICA.IETSI20MAY2020). Se cumplió con el principio de confidencialidad de los pacientes, recolectando los datos necesarios, después fueron anonimizados y los registros previos borrados. No se obtuvo consentimiento informado debido a que la fuente de datos fue secundaria (historia clínica y registros hospitalarios) y no hubo ninguna intervención con el paciente.

\section{RESULTADOS}

Entre marzo y mayo de 2020 se hospitalizaron 945 pacientes con COVID-19 (sospechoso o confirmado) en el hospital evaluado. Se revisaron 462 pacientes con PCR-RT positivo en hisopado nasofaríngeo, 58 fueron ambulatorios, 28 no correspondían a forma grave de enfermedad, 6 salieron de alta voluntaria y 25 tuvieron datos incompletos.

Se incluyeron 337 pacientes con enfermedad grave por COVID-19, fallecieron $215(63,8 \%)$ y egresaron del hospital con mejoría 122 (36,2\%). Fueron de sexo masculino 243 $(72,11 \%)$ y edad promedio de 63,59 años (DS 15,3, rango 23 y 99). Acudieron a la Emergencia refiriendo en promedio 7,4 días de enfermedad (DS 4,9, rango 1 y 35 ). 54,68\% registraron un tiempo de enfermedad mayor a 7 días. Habiéndose registrado disnea y fiebre como síntomas más frecuentes; $\mathrm{HTA}$, diabetes mellitus tipo 2 y obesidad como antecedentes más importantes (Tabla 1).

Tabla 1. Características clínicas de los pacientes con enfermedad grave por SARS-CoV-2 atendidos en un hospital de referencia, Lima Perú.

\begin{tabular}{|c|c|c|c|c|}
\hline \multirow{2}{*}{ Característica } & General & Fallece & Sobrevive & \multirow{2}{*}{$p^{a}$} \\
\hline & $(n=337)$ & $(n=215)$ & $(n=122)$ & \\
\hline Sexo masculino: $\mathrm{n}(\%)$ & $243(72,1)$ & $161(74,9)$ & $82(67,2)$ & 0,145 \\
\hline Edad: años promedio (DS) & $63,5(15,3)$ & $67,9(14,1)$ & $56,0(14,3)$ & $<0,001$ \\
\hline Hipertensión arterial: n(\%) & $130(38,6)$ & $98(45,6)$ & $32(26,2)$ & 0,006 \\
\hline Diabetes mellitus: n (\%) & $79(23,4)$ & $57(26,5)$ & $22(18,0)$ & 0,017 \\
\hline Obesidad: n (\%) & $77(22,9)$ & $52(24,2)$ & $25(20,5)$ & 0,533 \\
\hline $\begin{array}{l}\text { Tiempo de enfermedad: días } \\
\text { promedio (DS) }\end{array}$ & $7,4(4,9)$ & $7,3(5,3)$ & $7,7(4,2)$ & 0,985 \\
\hline Disnea: n (\%) & $244(85,6)$ & $153(84,1)$ & $91(88,4)$ & 0,487 \\
\hline \multicolumn{5}{|l|}{ qSOFA: $\mathrm{n}(\%)$} \\
\hline 0 & $16(4,9)$ & $6(2,8)$ & $10(8,8)$ & \\
\hline 1 & $189(58,2)$ & $120(56,9)$ & $69(60,5)$ & 0,343 \\
\hline 2 & $99(30,5)$ & $65(30,8)$ & $34(29,8)$ & 0,241 \\
\hline 3 & $21(6,5)$ & $20(9,5)$ & $1(0,9)$ & 0,009 \\
\hline \multicolumn{5}{|l|}{$\begin{array}{l}\text { Saturación de oxígeno al } \\
\text { ingreso: } \mathrm{n}(\%)\end{array}$} \\
\hline$>90 \%$ & $83(24,6)$ & $118(54,9)$ & $76(62,3)$ & \\
\hline $80-90 \%$ & $60(17,8)$ & $49(22,8)$ & $11(9,0)$ & 0,613 \\
\hline$<80 \%$ & $194(57,6)$ & $48(22,3)$ & $35(28,7)$ & 0,024 \\
\hline
\end{tabular}

a: Regresión de cox

Tabla 2. Resultados de exámenes auxiliares en pacientes con enfermedad grave por SARS-CoV-2 atendidos en un hospital de referencia, Lima Perú.

\begin{tabular}{|c|c|c|c|}
\hline $\begin{array}{c}\text { Exámenes auxiliares } \\
\text { Mediana (RIQ) }\end{array}$ & $\begin{array}{l}\text { Fallece } \\
(n=215)\end{array}$ & $\begin{array}{l}\text { Sobrevive } \\
(\mathrm{n}=122)\end{array}$ & Valor $\mathrm{p}^{\mathrm{a}}$ \\
\hline Proteína C Reactiva mg/dL & $20(18)$ & $14(14)$ & $<0,001$ \\
\hline Leucocitos $\mathrm{x}$ mm3 & $11220(6820)$ & $8560(5760)$ & $<0,001$ \\
\hline Linfocitos $x \mathrm{~mm} 3$ & $825(760)$ & $1040(645)$ & 0,002 \\
\hline Monocitos $\mathrm{x} \mathrm{mm3}$ & $515(370)$ & $470(320)$ & 0,416 \\
\hline Albúmina $\mathrm{g} / \mathrm{dL}$ & $3,47(0,60)$ & $3,70(0,66)$ & $<0,001$ \\
\hline Bilirrubina total $\mathrm{mg} / \mathrm{dL}$ & $0,67(0,43)$ & $0,56(0,37)$ & 0,011 \\
\hline Aspartato aminotransferasa $\mathrm{U} / \mathrm{L}$ & $57(39)$ & $57(48,5)$ & 0,816 \\
\hline Alanino aminotransferasa $\mathrm{U} / \mathrm{L}$ & $49(41)$ & $60(74)$ & 0,043 \\
\hline Deshidrogenasa láctica U/L & $456(217)$ & $375(157)$ & $<0,001$ \\
\hline Dímero $\mathrm{D}$ ug/mL & $2,30(6,78)$ & $1,19(1,94)$ & $<0,001$ \\
\hline Fibrinógeno mg/dL & $787,22(405)$ & $806,72(359)$ & 0,325 \\
\hline $\mathrm{PaO} 2 / \mathrm{FiO} 2 \mathrm{mmHg}$ & $92,50(109)$ & $194,75(175)$ & $<0,001$ \\
\hline Saturación arterial 02 \% & $91(7,0)$ & $93(5,0)$ & $<0,001$ \\
\hline Lactato $\mathrm{mmol} / \mathrm{L}$ & $1,9(1,0)$ & $1,5(0,8)$ & $<0,001$ \\
\hline \multicolumn{4}{|l|}{ Tomografía: } \\
\hline - Patrón Intersticial (\%) & $76(36,2)$ & $53(44,2)$ & $0,145 \mathrm{~b}$ \\
\hline - TSS c (rango 0-20) & $14(6)$ & $12(5)$ & $<0,001$ \\
\hline
\end{tabular}

Respecto a los exámenes de laboratorio, se encontraron diferencias entre el grupo de fallecidos y sobreviviente, entre los valores cuantitativos de: proteína $C$ reactiva (PCR) (valor $p<0,001)$, leucocitos (valor $p<0,001$ ), linfocitos (valor 
Tabla 3. Factores asociados a mortalidad en pacientes con enfermedad grave por SARS-CoV-2 atendidos en un hospital de referencia, Lima Perú.

\begin{tabular}{|c|c|c|c|c|c|c|c|c|}
\hline \multirow[t]{2}{*}{ Factores } & \multicolumn{2}{|c|}{ Fallecidos } & \multicolumn{2}{|c|}{ Sobreviviente } & \multirow{2}{*}{ Valor $\mathrm{p}^{\mathrm{a}}$} & \multirow{2}{*}{ HR } & \multicolumn{2}{|c|}{ IC $95 \%$} \\
\hline & $\mathrm{N}$ & $\%$ & $\mathrm{~N}$ & $\%$ & & & $\mathrm{LI}$ & LS \\
\hline Edad $>60$ años & 104 & 30 & 75 & 22 & $<0,001$ & 2,42 & 1,78 & 3,27 \\
\hline Antecedente HTAb & 99 & 29 & 33 & 10 & 0,006 & 1,52 & 1,13 & 2,06 \\
\hline Antecedente DM2c & 57 & 17 & 23 & 7 & 0,017 & 1,53 & 1,08 & 2,16 \\
\hline Compromiso pulmonar radiológico > 75\% & 87 & 26 & 19 & 6 & $<0,001$ & 1,84 & 1,40 & 2,43 \\
\hline Leucocitos $>14000 \times \mathrm{mm} 3$ & 66 & 21 & 18 & 6 & 0,018 & 1,43 & 1,06 & 1,91 \\
\hline Linfocitos $<800 \times \mathrm{mm} 3$ & 74 & 30 & 27 & 11 & 0,006 & 1,47 & 1,11 & 1,93 \\
\hline Proteína $\mathrm{C}$ reactiva $>20 \mathrm{mg} / \mathrm{dL}$ & 73 & 30 & 29 & 12 & 0,005 & 1,61 & 1,16 & 2,23 \\
\hline Albumina $<4,0 \mathrm{~g} / \mathrm{dL}$ & 176 & 62 & 72 & 25 & $<0,001$ & 2,89 & 1,61 & 5,19 \\
\hline Deshidrogenasa láctica > $350 \mathrm{U} / \mathrm{L}$ & 126 & 48 & 54 & 20 & 0,002 & 1,74 & 1,22 & 2,49 \\
\hline Dímero $\mathrm{D}>1,5 \mathrm{ug} / \mathrm{mL}$ & 102 & 41 & 35 & 14 & 0,005 & 1,61 & 1,16 & 2,23 \\
\hline Lactato $>1,5 \mathrm{mmol} / \mathrm{L}$ & 124 & 46 & 41 & 15 & 0,002 & 1,65 & 1,20 & 2,25 \\
\hline
\end{tabular}

a: Regresión de Cox, b: HTA hipertensión arterial, c: DM2 diabetes mellitus tipo 2

$p=0,002$ ), albumina (valor $p<0,001$ ), Bilirrubina total (valor $\mathrm{p}=0,011)$, deshidrogenasa láctica $(\mathrm{DHL})$ (valor $\mathrm{p}<0,001)$, dímero $\mathrm{D}$ (valor $\mathrm{p}<0,001), \mathrm{PaO2} / \mathrm{FiO2}$ (valor $\mathrm{p}<0,001$ ), Saturación de oxígeno (valor $p<0,001$ ) y Lactato (valor $\mathrm{p}<0,001)$. $\mathrm{Y}$ en relación al score de severidad tomográfica (TSS) se encontró diferencias significativas (valor $\mathrm{p}<0,001$ ). (Tabla 02).

Respecto al tratamiento, se empleó "fármacos contra COVID19 " en $74,5 \%$ de los pacientes, la asociación hidroxicloroquina mas azitromicina fue la más utilizada, administrándose en el $72 \%$ de los que posteriormente fallecieron y en el $79 \%$ de los que sobrevivieron ( $p=0,179)$. En cuatro casos se utilizó oseltamivir y en tres casos ivermectina.

Se administró corticoides en el $61,4 \%$ de casos que fallecieron y $44,3 \%$ de los que sobrevivieron $(p=0,154)$; el corticoide administrado fue metilprednisolona $49,0 \%$, hidrocortisona $5,9 \%$ y prednisona $0,3 \%$ del total de casos. La dosis de metilprednisolona fue de $80-500 \mathrm{mg}$ diarios, hidrocortisona 300 a $500 \mathrm{mg}$ diarios y prednisona $1 \mathrm{mg} / \mathrm{kg} / \mathrm{dia}$, entre 3 y 10 días de duración.

Se utilizó anticoagulación en $60,9 \%$ de los casos fallecidos y $42,62 \%$ de los sobrevivientes $(p=0,065)$, usando enoxaparina en $97,8 \%$ con una duración entre 3 a 14 días, a dosis profiláctica ( $1 \mathrm{mg} / \mathrm{Kg}$ de peso/ 24 horas) en el $66,7 \%$ del total de casos y en el 33,3\% restante anticoagulación plena (1 $\mathrm{mg} / \mathrm{Kg}$ de peso/12 horas). En 9 casos se utilizó heparina no fraccionada.

Se emplearon antibióticos en 320 pacientes $(94,96 \%$ del total), siendo los más utilizados inicialmente ceftriaxona $80 \%$, cefepime $9 \%$, pipercilina/tazobactam $8 \%$, meropenem $8 \%$, vancomicina $3,4 \%$. Iniciando con monoterapia en el $85 \%$ de casos.

En relación al soporte ventilatorio, 136 pacientes ingresaron a ventilación mecánica invasiva, $48,84 \%$ de los casos que posteriormente fallecieron y $25,41 \%$ de los sobrevivientes $(p=0,884) .35$ pacientes ingresaron a Unidad de cuidados intensivos $(\mathrm{UCl})(10,4 \%)$ los demás permanecieron en áreas críticas del servicio de emergencia. Luego de recibir tratamiento en salas de emergencia o $\mathrm{UCl}$, fueron a salas generales de medicina 55 pacientes $(16,32 \%$ del total). La mediana de hospitalización fue 10.32 días (entre 1 y 110 días).

Los factores asociados a mortalidad en el análisis bivariado (Tabla 3) fueron a) epidemiológicos: edad mayor de 60 años (HR: 2.415 IC95\%: 1.781-3.274), HTA (HR: 1.522 IC95\%: 1.1272.056) y diabetes mellitus (HR: 1.527 IC95\%: 1.079-2.161); b) exámenes auxiliares: leucocitosis > 14000 (HR: 1.427 IC95\%: 1.064-1.914), linfopenia < 800 (HR: 1.467 IC95\%: 1.1141.931), PCR > 20 (HR: 1.605 IC95\%: 1.155-2.230), albúmina < 4.0 (HR: 2.889 IC95\%: 1.608-5.189), Dimero D > 1.5 (HR: 1.608 IC95\%: 1.158-2.233), DHL > 350 (HR: 1.744 IC95\%: 1.2212.491), $\mathrm{PaO} 2 / \mathrm{FiO} 2<200$ (HR: 2.118 IC95\%: 1.475-3.041), lactato > 1.5 (HR: 1.645 IC95\%: 1.204-2.247), patrón radiológico TSS > 15 en tomografía (HR: 1.842 IC95\%: 1.398 2.427). No se encontró diferencia significativa entre los factores clínicos ni de tratamiento evaluados.

Tabla 4. Análisis multivariado de factores asociados a mortalidad en pacientes con enfermedad grave por SARSCoV-2 atendidos en un hospital de referencia, Lima Perú.

\begin{tabular}{lcccc}
\multicolumn{1}{c}{ Variable } & Valor $\mathrm{p}^{\mathrm{a}}$ & $\mathrm{HR}$ & \multicolumn{2}{c}{ IC 95\% } \\
\cline { 5 - 6 } & & & $\mathrm{Li}$ & $\mathrm{Ls}$ \\
\hline Edad (> 60 años) & 0,001 & 1,76 & 1,26 & 2,45 \\
Diabetes Mellitus tipo 2 & 0,007 & 1,63 & 1,14 & 2,32 \\
Compromiso tomográfico $(>75 \%)$ & $<0,001$ & 1,87 & 1,37 & 2,56 \\
Leucocitosis (> 14000) & 0,005 & 1,59 & 1,15 & 2,20 \\
Albumina $(<4,0 \mathrm{~g} / \mathrm{dL})$ & 0,006 & 2,49 & 1,30 & 4,75 \\
Lactato $(>1,5)$ & 0,001 & 1,77 & 1,25 & 2,49 \\
\hline a: Regresión de cox múltiple con método de seleción condiciona
\end{tabular}

a: Regresión de cox múltiple con método de selección condicional hacia adelante 
En el análisis de supervivencia multivariado (Tabla 4) se encuentran como factores asociados a mortalidad en COVID19 grave a: edad > 60 años (HR: 1,755 IC95\%: 1,256-2,453), diabetes mellitus tipo 2 (HR: 1,625 IC95\%: 1,140-2,318), leucocitos > 14000 (HR: 1,590 IC95\%: 1,150-2,200), albumina $<4,0$ (HR: 2,486 IC95\%: 1,302-4,746), lactato > 1,5 (HR: 1,766 IC95\%: 1,254-2,485) y compromiso pulmonar en tomografía (TSS > 15) (HR: 1,873 IC95\%: 1,370-2,561).

\section{DISCUSIÓN}

El presente estudio encontró como factores asociados a mortalidad en pacientes con COVID-19 grave, a la edad mayor de 60 años, la Diabetes mellitus tipo 2, mayor compromiso pulmonar, leucocitosis, hipoalbuminemia y lactato elevados al ingreso hospitalario. En el contexto de la etapa inicial de desarrollo de la pandemia en un país latinoamericano, con alto índice de contagio y elevadas tasas de mortalidad. Esto se diferencia de los factores de riesgo ya demostrados para desarrollar enfermedad grave por COVID-19.

La edad es un factor de riesgo demostrado para enfermedad grave y mortalidad, con un riesgo incrementado por cada año de incremento de la edad. Asimismo, la edad mayor a 60 años se asocia con un incremento de mortalidad en 1.90 veces; esto se explica por la disminución de mecanismos antiinflamatorios e incremento de los pro-inflamatorios con edad más avanzada ${ }^{(15-17)}$.

Los pacientes con diabetes mellitus desarrollan un curso clínico grave y tienen una mayor mortalidad, siendo considerado uno de los factores de riesgo más importantes ${ }^{(18)}$. Un meta-análisis que incluyó 16,000 pacientes, encontró que los diabéticos tienen 2,16 veces mayor probabilidad de desarrollar enfermedad grave por COVID-19 y el doble de probabilidades de morir debido a ella ${ }^{(19)}$. La diabetes y la infección por SARS COV-2, tienes vías patogénicas comunes, Dos de los receptores de coronavirus, la enzima convertidora de angiotensina 2 (ACE2) y la DPP4 también son transductores de las vías metabólicas que regulan la homeostasis de la glucosa, la fisiología renal, cardiovascular y la inflamación ${ }^{(19,20)}$.

La variación en la presentación de las imágenes tomográficas de acuerdo al tiempo de evolución de la enfermedad, guarda relación con el estado de gravedad. Se han definido 4 etapas: I (0-4 días) donde se encuentran opacidades en vidrio deslustrado, II (5-8 días) donde se encuentra un patrón de empedrado loco "crazy paving", III (9 a 13 días) donde se muestra consolidación y IV (más de 14 días) donde se aprecia una reducción gradual de la consolidación. Los pacientes recuperados de COVID-19 experimentaron la mayor gravedad de la enfermedad pulmonar en las tomografías obtenidas en el día 10 , es decir en la fase de consolidación ${ }^{(21)}$. Se ha demostrado la asociación entre porcentaje de compromiso pulmonar y severidad de la enfermedad, asociándose a mayor mortalidad $^{(13)}$.

En lo referente a leucocitosis, un meta-análisis que incluyó 6,320 pacientes procedentes de 62 artículos, encontró que los niveles elevados de leucocitos se asociaron a enfermedad severa (OR 2,62; IC 1,72-3,97), a una mayor probabilidad de ingreso a una UCI (OR 29,1, IC 2,9-291,8) y a una mayor mortalidad (OR 6,25, IC 2,05-19) $)^{(22)}$ probablemente relacionados a sobreinfección bacteriana.

Los niveles disminuidos de albúmina, hallazgo frecuente en los pacientes con forma grave de COVID-19, sugieren que la función sintetizadora del hígado está afectada significativamente; un meta-análisis de 45 estudios reporta que este parámetro de laboratorio se asocia a una mayor severidad de la enfermedad y a una peor evolución de los casos críticos $^{(23)}$.

El lactato elevado en nuestro estudio es un factor independiente asociado a mortalidad, similar lo reportado en hospitales de India y Alemania, donde los niveles elevados de lactato fueron predictores de mortalidad a las 24 horas de admisión a UCl y mortalidad en pacientes con COVID-19 $(p<0.01)$, esto se asocia a estados de hipoperfusión tisular y choque distributivo ${ }^{(24-27)}$.

La presencia de disnea es un factor asociado a enfermedad grave por COVID-19 ${ }^{(28,29)}$, sin embargo, algunos autores describen ausencia de disnea en injuria pulmonar severa, incluso en aquellos que fueron intubados e ingresados a ventilación mecánica, postulándose alteraciones a nivel del sistema nervioso central o las fibras C pulmonares responsables de la percepción de hipoxemia o por el shunt intrapulmonar debido a vasoplejía o fenómenos trombóticos intravasculares (hipoxemia silenciosa o "feliz") $)^{(31,32)}$.

La saturación periférica de oxígeno, se asocia a gravedad de enfermedad ${ }^{(17,33)}$, sin embargo no se encontró sigficancia en el presente estudio, quizás porque era un criterio de ingreso a hospitalización y al estudio. La presencia de 2 o más puntos de qSOFA no resultó significativo. Esto difiere de los reportes que encuentran asociación entre una mayor puntuación SOFA y mortalidad OR 1,30 (IC 1,11-1,53) por cada punto de incremento en China ${ }^{(34)}$ y $19 \%$ HR por un punto de incremento (IC 5-34) en España ${ }^{(16)}$. El uso de qSOFA se explica por su fácil aplicación al ingreso hospitalario, ya que no incluye resultados de exámenes auxiliares.

Otros factores clínicos y epidemiológicos (reportados como asociados a enfermedad grave y mortalidad en COVID-19) como sexo masculino ${ }^{(1,7)}$, tiempo de enfermedad mayor a 7 días, hipertensión arterial( ${ }^{(20,30)}$, obesidad y otras patologías cardiovasculares, resultaron no significativos en el presente estudio. Probablemente porque la mayoría de pacientes incluidos ya tenía este factor al haber desarrollado enfermedad grave.

La relación $\mathrm{PaO} 2 / \mathrm{FiO2}^{(16,24,25)}$ ha sido descrita como factor pronóstico en COVID-19, sin embargo, los valores iniciales evaluados, no muestran significancia. Otras variables de laboratorio como la linfopenia y elevaciones de proteína $C$ reactiva, deshidrogenasa láctica $(\mathrm{DHL})$ y dímero $\mathrm{D}$ se asociaron a formas severas de enfermedad y a una mayor mortalidad asociada ${ }^{(3,9,10,23)}$, en la presente investigación a pesar de tener asociación en el análisis bivariado, no resultaron significativas en el multivariado.

Al momento de la realización del trabajo no se tenía evidencia suficiente sobre el beneficio de los corticoides ni la dosis adecuada en la enfermedad grave por COVID-19, se 
dejaba a criterio clínico su indicación ${ }^{(35)}$. Las revisiones sistemáticas, que evaluaban el uso de metilprednisolona a dosis altas (mayores a $1 \mathrm{mg} / \mathrm{Kg} /$ día) habían incluido pocos pacientes, mencionaban que podría existir reducción la mortalidad (HR 0,41; IC 95\%: 0,20 a 0,83) ${ }^{(35,36)}$.

El estudio RECOVERY (Randomised Evaluation of COVid-19 thERapY) publicado en junio del 2020, realizado en más de 11500 pacientes de 175 hospitales del Reino Unido, demostró que el uso de $6 \mathrm{mg}$ diarios de dexametasona disminuyó la mortalidad en un tercio en pacientes con soporte ventilatorio (RR: 0,$64 ; 95 \% \mathrm{Cl}, 0,51$ to 0,81 ) y en un quinto en aquellos que recibieron suplemento de oxigeno ( $\mathrm{RR}: 0,82 ; 95 \% \mathrm{Cl}, 0,72$ to 0,94 ) de manera adicional se encontró que no hubo beneficio en aquellos pacientes que no requirieron oxigeno suplementario (RR 1,$19 ; 95 \% \mathrm{Cl}, 0,91$ to 1,55$)^{(37)}$. En nuestro estudio se emplearon dosis altas de corticoides, sin demostrar beneficio en la mortalidad.

La anticoagulación no demostró relación con mortalidad en COVID grave, sin embargo, es bien conocida la relación entre inflamación, hipoxia y el estado protrombótico reflejado en aumento de fibrina, los productos de degradación de la fibrina, el fibrinógeno y los dímeros $\mathrm{D}$, promovidos por la infección SARS-CoV- $2^{(38)}$. Así pues, también existen diversos estudios donde se observa el aumento de la incidencia de enfermedad tromboembólica a pesar de la anticoagulación profiláctica en sus diferentes presentaciones, Embolia pulmonar (EP) principalmente ${ }^{(39)}$ o trombosis venosa profunda $(T V P)^{(40)}$, estudios realizados principalmente en pacientes hospitalizados en UCI.

Diversos estudios han demostrado la necesidad y beneficio de la ventilación mecánica invasiva en pacientes con enfermedad grave por COVID-19 $9^{(3,10,12)}$, sin embargo son muchos los factores que influyen como el tiempo de evolución, la severidad y el equipamiento adecuado. En el presente estudio, a pesar de incrementarse el número de ventiladores mecánicos, no fueron suficientes para disminuir la mortalidad.

Entre las limitaciones del estudio esta recolección retrospectiva de datos y la falta de registro de índice de masa corporal en todos los pacientes (subregistro importante). Sólo se evaluaron los valores iniciales clínicos y de laboratorio, no se hizo seguimiento de los mismos. El colapso del sistema de salud obligó a adecuar ambientes de hospitalización y la falta de ambientes de cuidados críticos hizo que se amplíen las salas de emergencia para brindar soporte ventilatorio a muchos pacientes; sin embargo, los recursos no fueron suficientes para atender a todos los pacientes según su necesidad. La mayoría de pacientes acudían con más de 7 días de enfermedad (avanzada) por el aislamiento obligatorio instaurado o por la negativa de hospitalización a los pacientes que tenían saturación de oxígeno $>93 \%$ en el triaje de ingreso. Al inicio de la pandemia no estaban disponibles todos los medios diagnósticos para los pacientes, estos se fueron adaptando paulatinamente, de acuerdo al desarrollo de esta.

Finalmente podemos concluir que los factores asociados a mortalidad en pacientes con enfermedad grave por SARSCoV-2 en un hospital terciario de Perú son edad mayor de 60 años, el antecedente de diabetes mellitus tipo 2, compromiso pulmonar mayor a $75 \%$, leucocitosis al ingreso mayor a $14000 \times \mathrm{mm} 3$, albúmina menor de $4 \mathrm{~g} / \mathrm{dL}$ y lactato mayor de $1.5 \mathrm{mmol} / \mathrm{L}$.

\section{REFERENCIAS BIBLIOGRÁFICAS}

1. Yang $Y$, Xiao Z, Ye K, He X, Sun B, Qin Z, et al. SARS-CoV-2: characteristics and current advances in research. Virol $\mathrm{J}$. 2020;17(1):117. doi: 10.1186/s12985-020-01369-z

2. Nabil A, Uto K, Elshemy MM, Soliman R, Hassan AA, Ebara M, et al. Current Coronavirus (Sars-Cov-2) Epidemiological, Diagnostic and Therapeutic Approaches: an Updated Review Until June 2020. EXCLI J. 2020;19:992-1016. doi: 10.17179/excli2020-2554

3. Guan W-J, Ni Z-Y, Hu Y, Liang W-H, Ou C-Q, He J-X, et al. Clinical Characteristics of Coronavirus Disease 2019 in China. N Engl J Med. 2020;1-13. doi: 10.1056/NEJMoa2002032

4. Johns Hopkins University. Coronavirus Resource Center. COVID-19 Map.[Internet] 2020. [cited 2020 Sep 20]. Available from: https://coronavirus.jhu.edu/map.html

5. Ministerio de Salud - Perú. Sala situacional COVID-19 Perú [Internet]. Instituto Nacional de Salud. 2020 [cited 2020 Sep 20]. Available from: https://covid19.minsa.gob.pe/sala_situacional.asp

6. Acosta G, Escobar G, Bernaola G, Alfaro J, Taype W, Marcos C, et al. Caracterización de pacientes con COVID-19 grave atendidos en un hospital de referencia nacional del Perú. Rev Peru Med Exp Salud Publica. 2020;37(2):253-8. doi: 10.17843/rpmesp.2020.372.5437

7. Romano CM, Chebabo A, Levi JE. Past, present, and future of COVID19: a review. Braz J Med Biol Res. 2020;53(9):e10475. doi:10.1590/1414-431×202010475

8. Xu Z, Shi L, Wang Y, Zhang J, Huang L, Zhang C, et al. Pathological findings of COVID-19 associated with acute respiratory distress syndrome. Lancet Respir Med. 2020;2600(20):19-21. doi: 10.1016/S2213-2600(20)30076-X

9. Bhatraju PK, Ghassemieh BJ, Nichols M, Kim R, Jerome KR, Nalla AK, et al. Covid-19 in Critically IIl Patients in the Seattle Region - Case Series. N Engl J Med. 2020;382(21):2012-2022. doi: 10.1056/NEJMoa2004500

10. Grasselli G, Zangrillo A, Zanella A, Antonelli M, Cabrini L, Castelli A, et al. COVID-19 Lombardy ICU Network. Baseline Characteristics and Outcomes of 1591 Patients Infected With SARS-CoV-2 Admitted to ICUs of the Lombardy Region, Italy. JAMA. 2020;323(16):1574-1581. doi: 10.1001/jama.2020.5394

11. Wu C, Chen X, Cai Y, Xia J, Zhou X, Xu S, et al. Risk Factors Associated With Acute Respiratory Distress Syndrome and Death in Patients With Coronavirus Disease 2019 Pneumonia in Wuhan, China. JAMA Intern Med. 2020;180(7):1-11. doi: 10.1001/jamainternmed.2020.0994

12. Paranjpe I, Russak A, De Freitas JK, Lala A, Miotto R, Vaid A, et al. Clinical Characteristics of Hospitalized Covid-19 Patients in New York City. medRxiv Prepr Serv Heal Sci. 2020; doi: 10.1101/2020.04.19.20062117

13. Yuan M, Yin W, Tao Z, Tan W, Hu Y. Association of radiologic findings with mortality of patients infected with 2019 novel coronavirus in Wuhan, China. PLoS One. 2020;15(3):e0230548. doi: 10.1371 /journal.pone. 0230548

14. Valenzuela-Rodríguez GV, Rodriguez-Morales AJ, Mamani-Quiroz R, Ayala-García R, Pérez K, Sarmiento C, Calcino J, García-Carrión L, Amado-Tineo J. Cardiovascular risk factors and evolution of patients attended with COVID-19 in a National Reference Hospital from Lima, Peru. Rev Peru Investig Salud [Internet]. 3 de agosto de 2021 [citado 10 de diciembre de 2021];5(3):195-200

15. Zhou F, Yu T, Du R, Fan G, Liu Y, Liu Z, et al. Clinical course and risk factors for mortality of adult inpatients with COVID-19 in Wuhan, China: a retrospective cohort study. Lancet. 2020 Mar;395(10229):1054-62. doi: 10.1016/S0140-6736(20)30566-3

16. Rivera-Izquierdo $M$, Valero-Ubierna $M$, R-delAmo JL, FernándezGarcía MÁ, Martínez-Diz S, Tahery-Mahmoud A, et al. Sociodemographic, clinical and laboratory factors on admission associated with COVID-19 mortality in hospitalized patients: A retrospective observational study. PLoS One. 2020;15(6):e0235107. doi: 10.1371/journal.pone.0235107

17. Mejia F, Medina C, Cornejo E, Morello E, Vasquez S, Alave J, et al. Características clínicas y factores asociados a mortalidad en pacientes adultos hospitalizados por COVID-19 en un hospital público de Lima, Perú. ScieloPrepint. 2020; 
18. Rod JE, Oviedo-Trespalacios O, Cortes-Ramirez J. A brief-review of the risk factors for covid-19 severity. Rev Saude Publica. 2020; 54:60. doi: 10.11606/s1518-8787.2020054002481

19. Kumar A, Arora A, Sharma P, Anikhindi SA, Bansal N, Singla V, et al. Is diabetes mellitus associated with mortality and severity of COVID-19? A meta-analysis. Diabetes Metab Syndr Clin Res Rev. 2020;14(4):535-45. doi: 10.1016/j.dsx.2020.04.044

20. Lippi G, Wong J, Henry BM. Hypertension and its severity or mortality in Coronavirus Disease 2019 (COVID-19): a pooled analysis. Polish Arch Intern Med. 2020; 130(4):304-309. doi: 10.20452/pamw. 15272

21. Pan F, Ye T, Sun P, Gui S, Liang B, Li L, et al. Time Course of Lung Changes at Chest CT during Recovery from Coronavirus Disease 2019 (COVID-19). Radiology. 2020;295(3):715-21. doi: 10.1148 /radiol.2020200370

22. Elshazli RM, Toraih EA, Elgaml A, El-Mowafy M, El-Mesery M, Amin MN, et al. Diagnostic and prognostic value of hematological and immunological markers in COVID-19 infection: A meta-analysis of 6320 patients. PLoS One. 2020;15(8):e0238160. doi: 10.1371/journal.pone.0238160

23. Wu Y, Li H, Guo X, Yoshida EM, Mendez-Sanchez N, Levi Sandri GB, et al. Incidence, risk factors, and prognosis of abnormal liver biochemical tests in COVID-19 patients: a systematic review and meta-analysis. Hepatol Int. 2020; doi: 10.1007/s12072-020-10074-6

24. Kayina C, Haritha D, Soni L, Behera S, Nair P, Gouri M, et al. Epidemiological \& clinical characteristics \& early outcome of COVID19 patients in a tertiary care teaching hospital in India: A preliminary analysis. Indian J Med Res. 2020 Jul \& Aug;152(1 \& 2):100-104. doi: 10.4103/ijmr.IJMR_2890_20

25. Wendel Garcia PD, Fumeaux T, Guerci P, Heuberger DM, Montomoli J, Roche-Campo F, et al. Prognostic factors associated with mortality risk and disease progression in 639 critically ill patients with COVID19 in Europe: Initial report of the international RISC-19-ICU prospective observational cohort. EClinicalMedicine. 2020;25:100449. doi: 10.1016/j.eclinm.2020.100449

26. Wu M, Yao L, Wang Y, Zhu X, Wang X, Tang P, et al. Clinical evaluation of potential usefulness of serum lactate dehydrogenase (LDH) in 2019 novel coronavirus (COVID-19) pneumonia. Respir Res. 2020;21(1):171. doi: 10.1186/s12931-020-01427-8

27. Henry BM, Aggarwal G, Wong J, Benoit S, Vikse J, Plebani M, et al. Lactate dehydrogenase levels predict coronavirus disease 2019 (COVID-19) severity and mortality: A pooled analysis. Am J Emerg Med. 2020;38(9):1722-6. doi: 10.1016/j.ajem.2020.05.073

28. Zhang X, Cai H, Hu J, Lian J, Gu J, Zhang S, et al. Epidemiological, clinical characteristics of cases of SARS-CoV-2 infection with abnormal imaging findings. Int J Infect Dis. 2020;94:81-7. doi: 10.1016/j.ijid.2020.03.040

29. Rodriguez-Morales AJ, Cardona-Ospina JA, Gutiérrez-Ocampo E,
Villamizar-Peña R, Holguin-Rivera Y, Escalera-Antezana JP, et al. Clinical, laboratory and imaging features of COVID-19: A systematic review and meta-analysis. Travel Med Infect Dis. 2020;34:101623. doi: 10.1016/j.tmaid.2020.101623

30. Liang X, Shi L, Wang Y, Xiao W, Duan G, Yang H, et al. The association of hypertension with the severity and mortality of COVID-19 patients: Evidence based on adjusted effect estimates. J Infect. 2020;81(3):e44-7. doi: 10.1016/j.jinf.2020.06.060

31. Bertran Recasens B, Martinez-Llorens JM, Rodriguez-Sevilla JJ, Rubio MA. Lack of dyspnea in patients with Covid-19: another neurological conundrum? Eur J Neurol. 2020;27(9). doi: 10.1111/ene.14265

32. Dhont S, Derom E, Van Braeckel E, Depuydt P, Lambrecht BN. The pathophysiology of 'happy' hypoxemia in COVID-19. Respir Res. 2020;21(1):198. doi: 10.1186/s12931-020-01462-5

33. Galloway JB, Norton S, Barker RD, Brookes A, Carey I, Clarke BD, et al. A clinical risk score to identify patients with COVID-19 at high risk of critical care admission or death: An observational cohort study. J Infect. 2020;81(2):282-8. doi: 10.1016/j.jinf.2020.05.064

34. Liu J, Zhang S, Wu Z, Shang Y, Dong X, Li G, et al. Clinical outcomes of COVID-19 in Wuhan, China: a large cohort study. Ann Intensive Care. 2020;10(1):99. doi: 10.1186/s13613-020-00706-3

35. Ye Z, Wang Y, Colunga-Lozano LE, Prasad M, Tangamornsuksan W, Rochwerg B, et al. Efficacy and safety of corticosteroids in COVID-19 based on evidence for COVID-19, other coronavirus infections, influenza, community-acquired pneumonia and acute respiratory distress syndrome: a systematic review and meta-analysis. Can Med Assoc J. 2020;192(27):E756-67. doi: 10.1503/cmaj.200645

36. Callejas Rubio JL, Luna del Castillo JD, de la Hera Fernández J, Guirao Arrabal E, Colmenero Ruiz M, Ortego Centeno N. Eficacia de los pulsos de corticoides en pacientes con síndrome de liberación de citocinas inducido por infección por SARS-CoV-2. Med Clin (Barc). 2020;155(4):159-61. doi: 10.1016/j.medcli.2020.04.018

37. Dexamethasone in Hospitalized Patients with Covid-19 - Preliminary Report. N Engl J Med. 2020;NEJM2021436. doi: 10.1056/NEJMoa2021436

38. Han H, Yang L, Liu R, Liu F, Wu K-L, Li J, et al. Prominent changes in blood coagulation of patients with SARS-CoV-2 infection. Clin Chem Lab Med. 2020;58(7):1116-20. doi: 10.1515/cclm-2020-0188

39. Helms J, Tacquard C, Severac F, Leonard-Lorant I, Ohana M, Delabranche $X$, et al. High risk of thrombosis in patients with severe SARS-CoV-2 infection: a multicenter prospective cohort study. Intensive Care Med. 2020;46(6):1089-1098. doi:10.1007/s00134-02006062-x

40. Klok FA, Kruip MJHA, van der Meer NJM, Arbous MS, Gommers DAMPJ, Kant KM, et al. Incidence of thrombotic complications in critically ill ICU patients with COVID-19. Thromb Res. 2020;191:145-147. doi: 10.1016/j.thromres.2020.04.013. 